\title{
Can acupuncture help adults suffering from neuropathic pain? - A Cochrane review summary with commentary
}

\author{
Livia Puljak ${ }^{\mathrm{a}, \mathrm{b}, *}$ \\ ${ }^{a}$ Center for Evidence-Based Medicine and Health Care, Catholic University of Croatia, Zagreb, Croatia \\ ${ }^{\mathrm{b}}$ Agency for Quality and Accreditation in Health Care and Social Welfare, Zagreb, Croatia
}

Keywords: Acupuncture, neuropathic pain, Cochrane, systematic review

The aim of this commentary is to discuss in a rehabilitation perspective the published Cochrane review "Acupuncture for neuropathic pain in adults" by $\mathrm{Ju}$ et al. ${ }^{1}$, under the direct supervision of Cochrane Pain, Palliative and Supportive Care Group. This Cochrane Corner is produced in agreement with NeuroRehabilitation by Cochrane Rehabilitation.

\section{Background}

Neuropathic pain is challenging to treat due to its complex natural history, unclear etiology and insufficient response to standard physical therapy

\footnotetext{
*Address for correspondence: Livia Puljak, Center for Evidence-Based Medicine and Health Care, Catholic University of Croatia, Ilica 242, 10000 Zagreb, Croatia. Tel.: +385 21557 807; Fax: +385 21557 811; E-mails: livia.puljak@gmail.com; livia.puljak@unicath.hr.

${ }^{1}$ The abstract/plain language summary of this Cochrane review is taken from a Cochrane review previously published in the Cochrane Database of Systematic Reviews, 2017, Issue 12, DOI: 10.1002/14651858.CD012057.pub2. (see www.cochranelibrary.com for information). Cochrane reviews are regularly updated as new evidence emerges and in response to feedback, and Cochrane Database of Systematic Reviews should be consulted for the most recent version of the review.
}

interventions. A multimodal approach to neuropathic pain has been advocated, including combinations of pharmacological, physical, and cognitive interventions (Akyuz \& Kenis, 2014). Acupuncture is a traditional Chinese Medicine treatment modality that is increasingly popular in the Western world and some rehabilitation professionals may be trained in providing acupuncture treatment. For this reason, healthcare workers in the field of rehabilitation should be familiar with the current evidence about benefits and harms of acupuncture for various conditions. A Cochrane review addresses analgesic efficacy as well as safety of acupuncture for chronic neuropathic pain in adults (Ju et al., 2017):

\section{Acupuncture for neuropathic pain in adults}

(Ju, Wang, Cui, Yao, Liu, Zhou, Chen, \& Xia, 2017)

\section{What is the aim of the Cochrane Review?}

The aim of this Cochrane review was to analyze benefits and harms of acupuncture as an intervention for treatment of neuropathic pain in adults. 


\section{What was studied in the Cochrane Review?}

The population addressed in this review was adults with neuropathic pain conditions. The intervention studied was acupuncture, given alone or in combination with other interventions. Acupuncture therapy was defined as needle insertion and stimulation of somatic tissues for therapeutic purposes. The intervention was compared to sham acupuncture, treatment as usual or other active therapies. Primary outcomes studied were pain intensity measured with visual analog scale (VAS), pain relief defined as at least $30 \%$ pain relief and at least $50 \%$ pain relief over baseline. Secondary outcomes were any pain-related outcomes, withdrawals, adverse events (AE), serious AEs, specific AEs and quality of life (QoL).

\section{Search methodology and up-to-dateness of the Cochrane Review?}

The review authors searched for relevant studies up to 14 February 2017 using the Cochrane Central Register of Controlled Trials (CENTRAL), MEDLINE (Ovid), Embase (Ovid), four Chinese databases, clinical trials registries as well as the reference lists of included studies.

\section{What are the main results of the Cochrane Review?}

The review included 6 studies involving 462 adults suffering from chronic peripheral neuropathic pain. Five trials were conducted in China, and one in UK.

The review shows that:

- There are no clear benefits or harms of acupuncture for chronic neuropathic pain in adults in terms of pain intensity, pain relief and QoL, compared to sham acupuncture or other therapies.

- The review found small studies with very lowquality evidence and limited generalizability, as there were no studies conducted on younger adults, participants with different types of neuropathic pain, the use of diverse acupuncture techniques, and most importantly, the availability of clinically relevant outcomes.

- Evidence regarding safety of acupuncture was scarce.

\section{How did the authors conclude on the evidence?}

The authors concluded that there is insufficient evidence to either support or refute use of acupuncture treatment of neuropathic pain or a specific neuropathic pain condition.

\section{What are the implications of the Cochrane evidence for practice in neurorehabilitation?}

Acupuncture is considered to be one of the most popular complementary interventions and it is increasingly used for treatment of chronic pain. However, the Cochrane review of $\mathrm{Ju}$ et al. (Ju et al., 2017) about benefits and harms of acupuncture for neuropathic pain indicated that currently available evidence shows no clear difference between acupuncture treatment and tested comparators among adults with chronic neuropathic pain. Difference in pain intensity between manual acupuncture and sham acupuncture was minimal and based on one study only. For comparison of acupuncture with active therapies (mecobalamin combined with nimodipine, inositol), three trials were found. Data from those three studies show that there were fewer participants that did not have a clinical response in the manual acupuncture group, compared to the group treated with active therapies; however, those trials did not contain any other outcomes relevant for this Cochrane review. In two trials, participants who received a combination therapy of acupuncture with another active treatment versus those that received active treatment only (mecobalamin, and Xiaoke bitong capsule) had a modest decrease of pain intensity, but neither group achieved 'no worse than mild pain'.

Data on quality of life were limited and showed no clear difference between the study groups. Additionally, the reviewers concluded that there is a lack of evidence on safety of acupuncture, which precludes a more comprehensive evaluation of benefit and harm.

All identified trials analyzed only manual acupuncture. There were no trials that investigated other types of acupuncture treatments, such as electroacupuncture, warm needling, or fire needling. None of the studies compared acupuncture with treatment as usual.

Rehabilitation professionals should be aware that this Cochrane review did not identify clear benefits or harms of acupuncture for treating chronic 
neuropathic pain in adults. Present evidence comes from several small trials with very low-quality evidence, which included participants with peripheral neuropathic pain, mostly with diabetic neuropathy, further limiting applicability of these findings to various clinical contexts. Because of risk of bias and low quality of evidence, Cochrane authors highlighted that they have very little confidence in the effect estimate and that the true effect is likely to be substantially different from the estimate of effect presented in this review, based on the six identified studies. This is important information for practice and research due to the concerns regarding the robustness of the current evidence, and the potential that future studies may generate different conclusions. The message for rehabilitation professionals is that the evidence-base about this intervention is insufficient to draw sound conclusions and that there is a need for conducting further, better quality research on this topic to obtain more conclusive evidence one way or another.

Cochrane authors did find five studies that are still ongoing (three from the USA, one from Hong Kong and one from Korea), and eight studies that they classified as awaiting classification (five from China and the rest from Germany, Spain and USA) due to lack of information that precludes including them into analysis or excluding them. When available, data from those trials may inform future practice and provide conclusive information for practitioners.

\section{Acknowledgments}

The author thanks Cochrane Rehabilitation and Cochrane Pain, Palliative and Supportive Care Group for reviewing the contents of the Cochrane Corner.

\section{Conflict of interest}

The author declares no conflicts of interest.

\section{References}

Akyuz, G. \& Kenis, O. (2014). Physical therapy modalities and rehabilitation techniques in the management of neuropathic pain. American Journal of Physical Medicine \& Rehabilitation, 93(3), 253-259. doi: 10.1097/PHM.0000000000000037

Ju, Z.Y., Wang, K., Cui, H.S., Yao, Y., Liu, S.M., Zhou, J., Chen, T.Y., \& Xia, J. (2017). Acupuncture for neuropathic pain in adults. Cochrane Database of Systematic Reviews, Issue 12, CD012057. doi: 10.1002/14651858.CD012057.pub2 